\title{
EXPERIMENTAL AND NUMERICAL STUDY OF HEAT TRANSFER PERFORMANCE FOR AN ENGINE REPRESENTATIVE TWO-PASS ROTATING INTERNAL COOLING CHANNEL
}

\author{
Zhi Wang \\ School of Aeronautics and Space \\ Universidad Politecnica de Madrid \\ Madrid 28040, Spain \\ Email: zhi.wang@upm.es
}

\author{
Roque Corral* \\ Advanced Engineering Direction \\ Industria de TurboPropulsores S.A. \\ 28830 Madrid, Spain \\ Email: roque.corral@itp.es
}

\author{
Francois Chedevergne \\ ONERA \\ 29 Avenue de la Division Leclerc \\ 92322 Chatillon Cedex, France \\ Email: francois.chedevergne@onera.fr
}

\section{ABSTRACT}

This paper investigates, both experimentally and computationally, the heat transfer performance on an engine representative varying aspect ratio two-pass internal cooling channel, in both stationary and rotating conditions. The test geometry and design parameters were suggested by SNECMA as a representative HPT blade two-pass internal cooling channel. The cooling channel has radially outward flow in the first passage with an aspect ratio of 1:2.25 and after a 180 degree sharp turn, a radially inward flow in the second passage with an aspect ratio of 1:1.85. One side of the two passages is equipped with 45 degree angled rib turbulators with a rib spacing $P / e=7$ and blockage ratio $e / D_{h}=0.116$. The other side is smooth in order to have optical access for experiment.

The experiment was performed at three Reynolds numbers: $15,000,25,000$, and 35,000. Both forward and backward rotating directions were tested in order to study the heat transfer performance of the ribbed surface as trailing wall or leading wall individually. The tested Rotation numbers were $\mathrm{Ro}= \pm 0.3$ at $\operatorname{Re}=15,000$ and $\operatorname{Re}=25,000$, whereas the Rotation number was reduced to \pm 0.22 at $R e=35,000$, due to restrictions of the test facility. Infrared thermography technology is used to capture the temperature field for further evaluation of heat transfer performance.

\footnotetext{
*Also Associate Professor at the Department of Fluid Mechanics and Aerospace Propulsion of the School of Aeronautics and Space, UPM, Madrid 28040, Spain
}

Numerical simulations for all experimental cases were conducted using the same geometry including the air feeding system, applying the experimental wall temperature distribution in order to properly capture inlet and buoyancy effects, with the $k-\omega-S S T$ turbulence model. Numerical results show overall agreement and similar trends than the experimental data. Numerical results also show that the rotation effects alter the internal flow significantly, resulting in different surface heat transfer distributions. Particularly, it is shown that heat transfer performance of the pressure side is not enhanced by the rotation in this study, which is a surprising result. This behavior was captured both in the experiments and the numerical predictions.

\section{NOMENCLATURE}

$\begin{array}{ll}A= & \text { area } \\ A R= & \text { channel aspect ratio: width-to-height } \\ B o= & \text { Buoyancy number, } B o=R o^{2} \frac{r}{d_{h}} \frac{\left(T_{w}-T_{\text {ref }}\right)}{T_{\text {ref }}} \\ C_{p}= & \text { specific heat capacity } \\ d_{h}= & \text { hydraulic diameter } \\ e= & \text { rib height } \\ e / d_{h}= & \text { blockage ratio: rib height-to-hydraulic diameter } \\ h= & \text { heat transfer coefficient } \\ \dot{m}= & \text { mass flow rate } \\ N u= & \text { Nusselt number } \\ k= & \text { thermal conductivity } \\ P / e= & \text { rib spacing: pitch-to-height }\end{array}$




$\begin{array}{ll}\operatorname{Pr}= & \text { Prandlt number } \\ Q= & \text { heat } \\ \operatorname{Re}= & \text { Reynolds number, } R e=U_{0} d_{h} / v \\ \operatorname{Ro}= & \text { Rotational number, } R o=\Omega d_{h} / U_{0} \\ s= & \text { curvilinear coordinate } \\ T_{r e f}= & \text { reference temperature } \\ T_{w}= & \text { wall temperature } \\ U_{0}= & \text { inlet mean velocity } \\ \alpha= & \text { effective heat factor }\end{array}$

\section{INTRODUCTION}

The continuous efforts for improving efficiency and thermal performance in modern aero-engines lead to the use of very high turbine entry temperatures and to minimize the amount of coolant flow consumption. Therefore, internal cooling techniques of gas turbine rotor blades play an extremely important role in the turbine design and development process. In order to enhance the heat transfer performance of internal cooling systems, complex internal cooling channels are cast in the blades. Heat transfer enhancement devices such as ribs, pin-fins or dimples are also used to promote turbulence development and increase heat transfer efficiency. One of the most typical and widely studied configuration to enhance heat transfer consists of ribbed channels connected by sharp 180 degree bends.

There are many factors affecting the level of heat transfer enhancement in ribbed channels. For instance, depending on the internal location of the channels inside the rotor blade, its aspect ratio (width-to-height ratio) can vary within a wide range, typically between 1:4 to 4:1. It has been shown that the heat transfer and fluid flow field have a strong dependence on the channel aspect ratio. Furthermore, the rib arrangement inside the channel, such as the orientation, spacing, or blockage ratio have a significant impact on the flow passing through the channel and therefore, the heat transfer enhancement. The advantage of using rib turbulators is that the rib promoted separation - reattaching flow increases the mixing between the wall boundary layer and the mainstream, enhancing the heat transfer level at surface. Meanwhile, ribs only disturb the near wall regions, minimizing the pressure drop penalty.

Many experimental studies, have been conducted to understand the effects of channel aspect ratio and different rib parameters in heat transfer efficiency. Han [1] studied the heat transfer behavior on five channels with different aspect ratio $(A R=1: 4,1: 2$, 1:1, 2:1, 4:1). Then Han and Park [2] and Park et al. [3] studied the effect of rib orientation. Generally speaking, these studies concluded that low aspect ratio channels have higher cooling efficiency than high aspect ratio channels. For low aspect ratio channels, the 45/60 degree angled ribs provide the highest heat transfer performance, while 30/45 degree angled ribs are better suited for high aspect ratio channels. Taslim and Wardsworth
[4] and Taslim and Lengkong [5] extended the study to the heat transfer performance on the rib surface for three different rib spacing $(P / e=5,8.5,10)$ and blockage ratio $\left(e / d_{h}=0.133\right.$, $0.167,0.25)$. They suggested that the heat transfer enhancement on the rib surface is fairly insensitive to the rib height, whereas the pressure drop increases substantially with it, and as a result the former configuration if favor over the latter. In the work done by Taslim and Korotky [6], they further concluded that for low blockage ratios, the result of $P / e=10$ has the highest heat transfer coefficient. For high blockage ratio ribs, the results for heat transfer were insensitive to the $P / e$ ratio.

There has been an increasing interest in heat transfer performance studies for rotating channels. Subject to rotation, Coriolis forces induce secondary flows and vortices in transverse direction along the channel. In a ribbed channel, Coriolis forces interact with the separated flow promoted by a variety of turbulent devices producing a large diversity of secondary flows, that characterize their corresponding heat transfer performance. Therefore, the interaction of the secondary flow induced by rotation and the rib turbulators results in different heat transfer patterns than in stationary channels.

Wagner er al. [7, 8] conducted a detailed experimental study to determine the effect of rotation on the local heat transfer of a multipass square channel with smooth walls. They concluded that the first pass of the coolant passage created a thinner boundary layer on the trailing surface than in the leading surface resulting in a increased and decreased, respectively, heat transfer. In the second pass, the leading surface heat transfer was higher than the trailing surface because of the reversal of the Coriolis force direction.

Johnson et al. [9] used their square channel to measure the heat transfer coefficient in $45 \mathrm{deg}$ angled ribbed channels. Similar to their smooth channel counter part, the leading and trailing surface of the channel experienced different levels of heat transfer enhancement. However, because the rib turbulators increased the heat transfer at the channel walls, the difference between the leading and trailing ribbed wall was smaller than that of the smooth walls. Similar results have been observed in square channels with angled ribs by Dutta ad Han [10] .

Rotation effects on the heat transfer enhancement of ribbed channels have been studied in a wide range of aspect ratios as well. Al-Hadhrami and Han [11] and Fu et al. [12] investigated the heat transfer enhancement in $A R=2: 1$ channels with rib turbulators. Even higher aspect ratio, up to $4: 1$, have been the focus of several studies as well. With these channels having relatively wide leading and trailing surfaces, both studies report significant spanwise variation in the heat transfer coefficient on both sides. However, the effect of rotation is more significant in low aspect ratio than in high aspect ratio channels. When the aspect ratio is reduced from 1:2 to 1:4, Fu et al. [13] showed that the combination of the rib and rotation induced secondary flow can adversely 
affect the heat transfer from the first pass leading surface.

Furthermore, the interest have been widely spread to other details over recent years. Cases with differing inlet and outlet cross sections were investigated by Iacovides et al. [14] and Lucci et al. [15] on a smooth channel with 180 degrees bend, a square section in the first passage and $A R=2: 1$ in the second passage, both experimentally and numerically. They found that a larger cross section leads to a decelerated flow in the second passage, resulting in a change of the separation-reattachment flow pattern and heat transfer level after the turn, compared to that seen in a constant area section.

Inlet shape effects have been studied by Wirght et al. [16], they pointed out that a developing entrance condition enhanced the heat transfer compared to that of a fully developed flow, and that increasing the rotation number decreases the effect of the entrance. Huh et al. [17] also confirmed that a sudden expansion entry shape can increase the heat transfer in the first pass of a two-pass channel. These findings are also important for numerical validation, suggesting that the entry geometry should be taken into consideration in the simulations.

All these complications inevitably result in significantly different flow fields and heat transfer behaviors. Although many studies have been focused on the heat transfer in a variety of configurations, most of the data cover just a relatively narrow range due to the different research targets of different institutions. Moreover, engineering designs have to deal with different types of constrains such as geometry, structure, pressure drop penalty, leading to the use of a combination of factors during the cooling channel design. With so many factors under consideration, the designer can not simply apply the idealized knowledge to conduct the actual design process. Therefore, research oriented to the use of models under design constrains, and the validation of sophisticated numerical tools is necessary to address the design of internal cooling channels in realistic turbine rotor blades.

Computational Fluid Dynamics (CFD) has significantly expanded its footprint during the last two decades in the design and analysis of turbomahinery components, and is routinely used in the aerodynamic and aeromechanic analysis of compressor and turbine blading. However its penetration in the analysis of cooled rotor blades is much smaller than in the aforementioned cases. In spite of the vast improvements of CFD methods and computer power during the last 30 years, numerical methods have yet nowadays well known shortcomings. Concerning cooled rotor blades, the limitations have been mostly related with the capability to agile meshing of engine representative configurations [18] and obtain credible solutions within the design loop of the gas turbine companies. Most of aerodynamic analyses of compressors and uncooled turbines are RANS based, and hence are far from perfect. Analogously, turbulence modeling of cooled rotor blades is not ideal either. However at industrial level what is important is to assess and calibrate the limitations of existing tools in realistic configurations to leverage CFD methods in industrial environment. This paper is placed within this context.

Meanwhile, a lot of numerical research is conducted, in parallel with heat transfer experiments, to address the deficiencies of RANS based approaches using LES and for rotating channels. However, although these techniques will compete in the near future with classical experiments, their cost is several orders of magnitude larger than that of RANS methods, and will struggle yet for some years to get introduced in complex design loops where a fast turnaround time is mandatory, and CFD is not really used as the primary design tool. This work is classed in this environment and aims to deliver the reader the information of which is the level of accuracy that can be expected using RANS based methods on an engine representative geometry to further proceed to their calibration, With this aim in mind, quick analysis methods need to be combined with complex configurations.

Numerous papers can be found to numerically simulate fluid flow and heat transfer in a two-pass channel with 180 degree bend. Numerical simulations provide details which are difficult to obtain by experiments. Lin et al. [19] investigated the effect of 45 degree angled ribs on the heat transfer coefficients in a rotating two pass square channel using low Reynolds number $k-\omega$ model. They found that the angled ribs create an asymmetric heat transfer behavior after the ribs. This is because the angled ribs itself developed a secondary flow in spanwise direction. The flow and heat transfer through a two pass smooth and 45 degree rib-roughened rectangular duct has been reported by Al-Qahtani et al. [20, 21] using a Reynolds stress turbulence model. They found reasonable match with experiments, although in certain regions there are significant discrepancies. Lucci et al. [15] used low Reynolds number $k-\varepsilon$, no linear $k-\omega$, and Reynolds stress to study the heat transfer performance in rotating square U-bend channel. Shevchuk et al. [22] performed numerical validations for a varying aspect ratio two-pass internal cooling. In general, simulations predicted the main features of heat transfer behavior within around a $15 \%$ deviation with respect the experiments, however, depending on geometry parameters or particular areas of the channel, the differences could rise up to $50 \%-100 \%$.

Numerical simulation of internal cooling flows is difficult, because there is too much mixing due to large eddies coming from separation and reattachment due to the ribs and other geometric entities, and secondary flows induced by rotations. Bonhoff et al. [23, 24] show the limitations of turbulence models in angled rib duct flow. The accuracy of RANS solvers in internal cooling problems rely on the assumption of turbulence models. For instance, the second moment closure models resolve the near wall flow all the way to the solid wall rather than using the loglaw assumption in the viscous sublayer compared to the simpler isotropic eddy viscosity models. Thus, the results are more accurate. The $k-\omega$ model can predict reasonable well this type of flows in many cases due to the fact that the turbulence dissi- 
pation is specified in terms of the turbulent kinetic energy in the near wall region and does not require the use of wall functions provided that fine enough grids are used closed to wall [25].

With the rapid growth of computer power, LES is also used to investigate complex flow features with high fidelity in internal cooling problems. Sewall and Tafti [26] performed LES simulation in the 180 bend region of a stationary ribbed channel. They discovered the high gradients of heat transfer augmentation on the back wall and downstream outside wall, where mean flow impingement occurs. Abdel-Wahab and Tafti [27] performed LES studies to investigate the flow features with stationary channel with inclined ribs. Their LES results show secondary flow details in angle ribbed channels, which are differ from 90 degree ribs, also very accurate predictions in both flow-field and heat transfer performance were obtained. Saha and Acharya [28] reported the comparison of URANS with LES, they concluded that URANS predictions reasonable well with measurements, and LES is even better. Fransen et al. [29] used LES to simulate a ribbed rotating channel and also obtain very good agreement in aerodynamic data and heat transfer predictions as well against experimental data .

Although LES can provide more accurate flow and heat transfer predictions than RANS, the computational time limits its use in practical designs. For instance, the LES references listed above, either used only a small fraction of the model with periodic boundary conditions or tested just a single case, which is beneficial for flow physics and detail analysis. However, full scale model tests or systematic parametric studies are not affordable using LES. In industrial design process, it would be desirable to use RANS simulations that are able to capture the dominant flow features within reasonable turnaround times.

This paper is one of the few that study both, experimentally and numerically, the heat transfer behavior on an engine representative varying aspect ratio two-pass channel with 45 degree ribs with a combination of non-idealized parameters, to establish an industrial level experimental data base and enhance the understanding in practical aspects of turbine rotor blade design. Furthermore, it is a key piece of work to validate numerical tools against experimental results in an engineering environment with so many combination factors, to assess and calibrate the limitations and the level of accuracy that can be expected using RANS based method on an engine representative geometry.

\section{EXPERIMENTAL METHOD}

\section{Test Setup}

The experimental campaign was conducted at ONERA BATHIRE rig facility, which is used for the testing of both, sta-

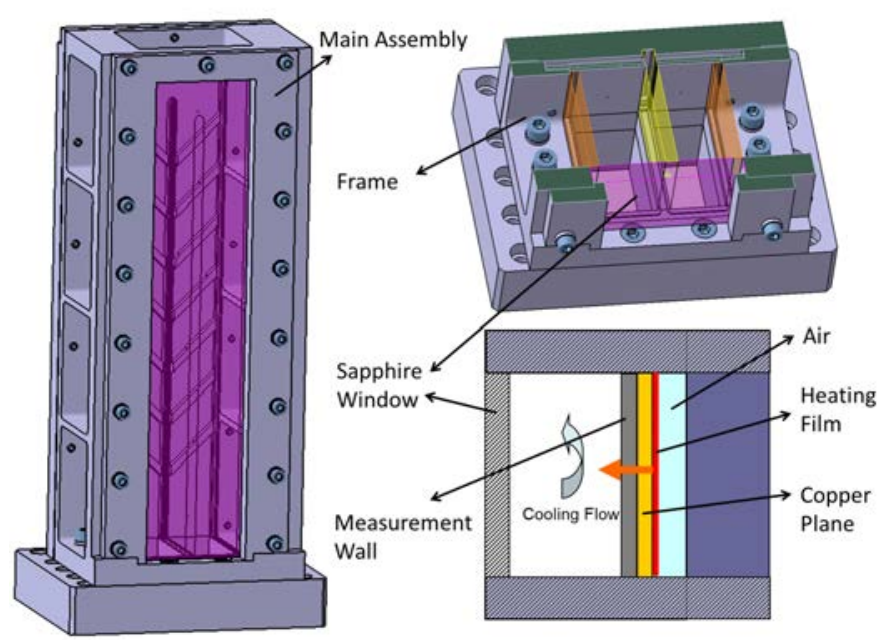

FIGURE 1. MAIN TEST SECTION OF EXPERIMENTAL MOCK-UP

tionary and rotating internal cooling systems. The main test section of the experimental mock-up is shown in Fig. 1.

The assembly provides structural support and ensures the strength of the whole mock-up. It connects to the rig shaft through a flange which enables the air-supply and the routing of thermocouples and electrical supply for the heating system. The frame is fixed to the assembly. It clamps the lateral windows and the separation wall between the two passages. In front of the measurement wall, there is a window made up of synthetic sapphire, that is transparent in the infrared range, to enable an optical access for the infrared camera.

The measurement wall is fixed on the frame with no contact with the assembly in order to prevent backward thermal conduction losses. The measurement wall consists of three layered components. A stainless steel plate whose surface is in contact with the cooling flow stream. This face is black color painted in order to reach a emissivity close to 1 . It is equipped with 5 thermocouples for temperature calibration and measurement. A copper plate, on which the stainless steel plate is brazed, is used to uniform the heat flux of the stainless steel plate. At last, the heating film is glued to the other side of the copper plate. The measurement plate features a cut-out in which the separating wall is fitted. This cutting prevents lateral conduction between the two branches.

Infrared thermography measurements are made when the mockup has reached its thermal equilibrium. Monitoring and data acquisition systems enable to follow in real time the evolution of the thermocouples installed on the measurement plate. On the PC screen, inlet and outlet flow temperatures are also displayed. These graphs allow to estimate when the thermal equilibrium has been reached. The test sequence is: (i) Flow injection at a flow rate corresponding to the target Reynolds number. The flow rate is regulated by the monitoring program. (ii) Heating power 


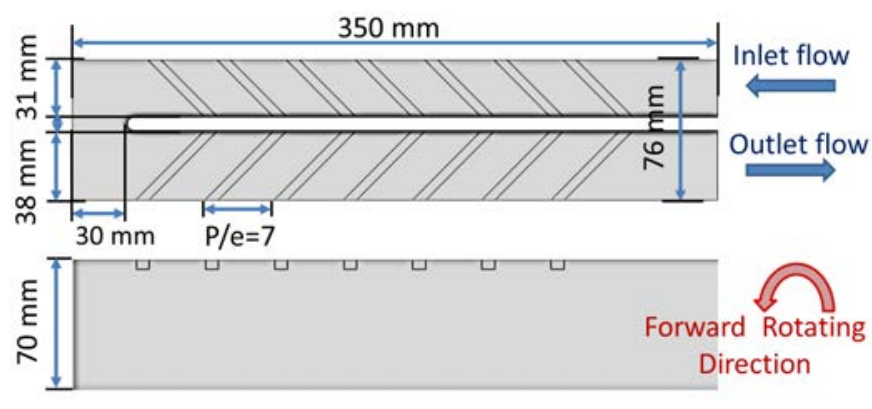

FIGURE 2. COOLING CHANNEL SKETCH

adjustment by changing the supply voltage for the heating film. (iii) Waiting for the thermal equilibrium and (iv) Recording from the infrared camera. A synchronization mechanism between the camera and the rotating arm is implemented to trigger properly the recording.

\section{Channel Model}

The testing geometry was designed by SNECMA based on a HPT rotor blade two-pass internal cooling channel. It consists of two rectangular channels connected by a 180 degree sharp turn. The overall dimensions of the test channel can be found in Fig. 2. The total length of the channel is $350 \mathrm{~mm}$, and the height 70 $\mathrm{mm}$. The width of the upstream and downstream passages is 31 $\mathrm{mm}$ and $38 \mathrm{~mm}$, respectively. The gap between the two channels is $7 \mathrm{~mm}$ and the distance between the divider wall and outer end wall is $30 \mathrm{~mm}$. The outward upstream passage has an aspect ratio of $A R=1: 2.25$ and after the turn, the inward downstream passage has an aspect ratio $A R=1: 1.85$. One side of the two channels is equipped with 45 degree square cross section skewed rib turbulators, with a rib spacing $P / e=6$ and a blockage ratio $e / d_{h}=0.116$, where the hydraulic diameter is referred to the upstream passage. In total 14 ribs are arranged in parallel, while the other side remains smooth in order to ease the optical access.

\section{Data reduction}

This study investigates the heat transfer behavior in stationary and rotating conditions. Three Reynolds numbers have been tested: $\operatorname{Re}=15,000,25,000$ and 35,000. At each Reynolds number, three tests were conducted: stationary; forward rotation direction $(\operatorname{Ro}>0)$, where the ribbed surface behaves as the trailing wall (pressure side) of a rotor blade; and backward rotation direction $(\operatorname{Ro}<0)$, where the ribbed surface behaves as leading wall (suction side). The mean buoyancy number, $B o$ is about 0.15, based on the mean radius of the channel and mean wall temperature which is similar to that found in other works. Locally the $B o$ can vary between $0.12-0.2$, depending on surface heating level and radial location.

\begin{tabular}{|c|c|c|c|}
\hline Re & RPM & Ro & Heating Power (W) \\
\hline \hline 15,000 & 384 & $0, \pm 0.3$ & $100,150,200$ \\
\hline 25,000 & 587 & $0, \pm 0.3$ & $100,150,200$ \\
\hline 35,000 & 600 & $0, \pm 0.22$ & $100,150,200$ \\
\hline
\end{tabular}

TABLE 1. EXPERIMENTAL TEST MATRIX

The test matrix is shown in Tab. 1, where the Reynolds and Rotation numbers are based on the hydraulic diameter of the inlet passage.

In order to determine the effective heat transferred in the measurement wall, calibration tests were performed in advance with the same geometry but with a smooth channel at each Reynolds number and rotating condition. Wall temperature and reference temperature were measured at a location where the heat transfer coefficient had been determined by previous studies. Because of the use of a highly conductive copper plate next to the measurement wall, heat flux of the measurement wall is considered spatially uniform in the tests. Then, the net heat $Q_{n e t}$ and effective heat factor, $\alpha$, can be calculated in each calibration test as

$$
Q_{n e t, c}=A h_{c}\left(T_{w, c}-T_{r e f, c}\right)=\alpha Q
$$

where $A$ is the area of the measurement wall, and $Q$ is the total heating power. Three heating powers: $100 \mathrm{~W}, 150 \mathrm{~W}$ and $200 \mathrm{~W}$ are used to smooth the error of a single test. The obtained $\alpha$ values are then used for the ribbed cases assuming that the losses remain identical under same Reynolds number and rotating conditions for the same channel geometry. The evaluation of heat transfer coefficient is done with the effective heating power $\alpha Q$ and wall temperature obtained by IR measurements as:

$$
\begin{cases}h=\frac{1}{3} \sum_{i=1} h_{i} & , i \in[1,2,3] \\ h_{i}=\frac{\alpha_{j} Q_{j} / A-\alpha_{k} Q_{k} / A}{\left(T_{w, j}-T_{r e f, j}\right)-\left(T_{w, k}-T_{r e f, k}\right)} & , \forall i \neq j \neq k \\ T_{\text {ref }, i}=T_{i n}+s \cdot \frac{\alpha_{i} Q_{i}}{C_{p} \cdot \dot{m}} & \end{cases}
$$

where $C_{p}$ is the specific heat capacity, $\dot{m}$ the mass-flow rate, and $s \in[0,1]$ stands for the curvilinear coordinate of the current location along the center line of the channel. The reference temperature at any location of the test section is calculated based on the conservation of mean energy. 

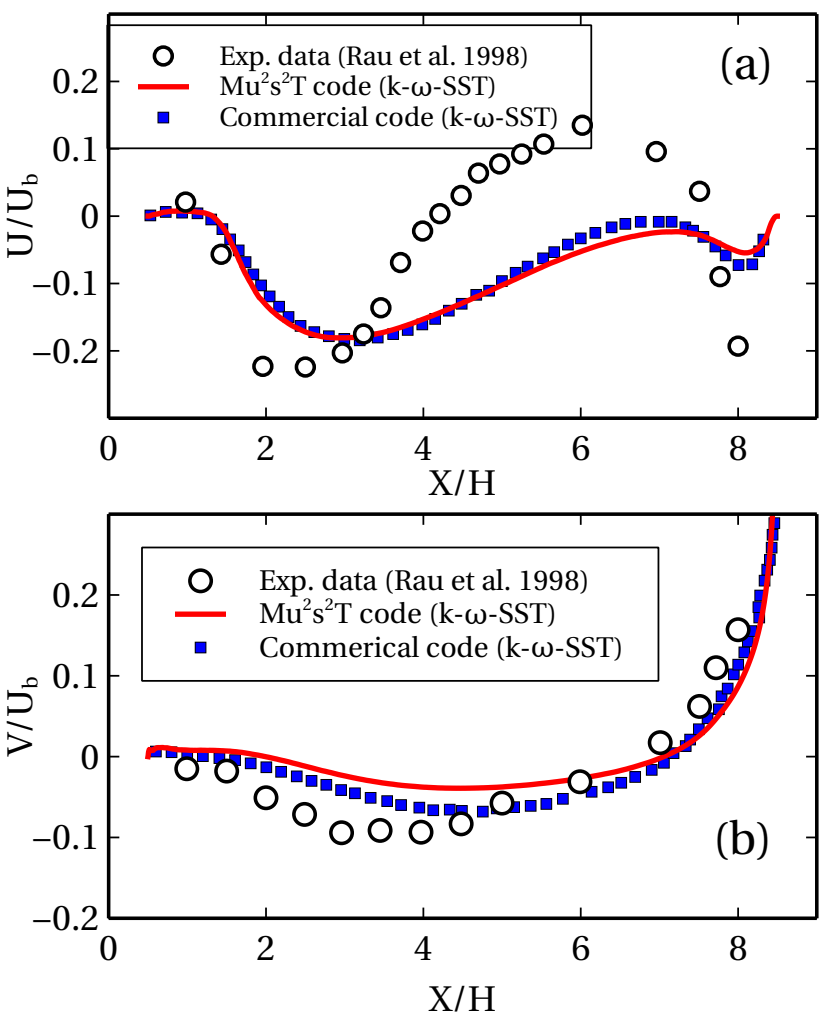

FIGURE 3. STREAMWISE VELOCITY PROFILES CLOSE TO WALL (A) AND VERTICAL VELOCITY PROFILE AT RIB HEIGHT (B) [31]

Consider the effective resolution of the infrared camera, and the potential non-uniformity of actual heat flux in measurement wall, for providing more reliable data, the final experimental results are provided as section averaged Nusselt number in the area comprised between two ribs, normalized with the Nusselt number obtained from the Dittus-Boelter correlation, $N u_{0}$ [30]. This correlation is used for fully developed turbulent flow through a smooth stationary pipe. Therefore, the $N u / N u_{0}$ ratio is given as:

$$
\frac{N u}{N u_{0}}=\left(\frac{h \cdot d_{h}}{k}\right) \frac{1}{0.023 R e^{0.8} \operatorname{Pr}^{0.4}}
$$

where heat transfer coefficient $h$ is obtained by Eq. 2 and $d_{h}$ is the hydraulic diameter of inlet passage.

\section{NUMERICAL METHOD}

Several attempts have been previously performed to simulate the fluid flow and heat transfer in a two-pass channel with a 180 degree turn. It is widely admitted that that RANS equations with

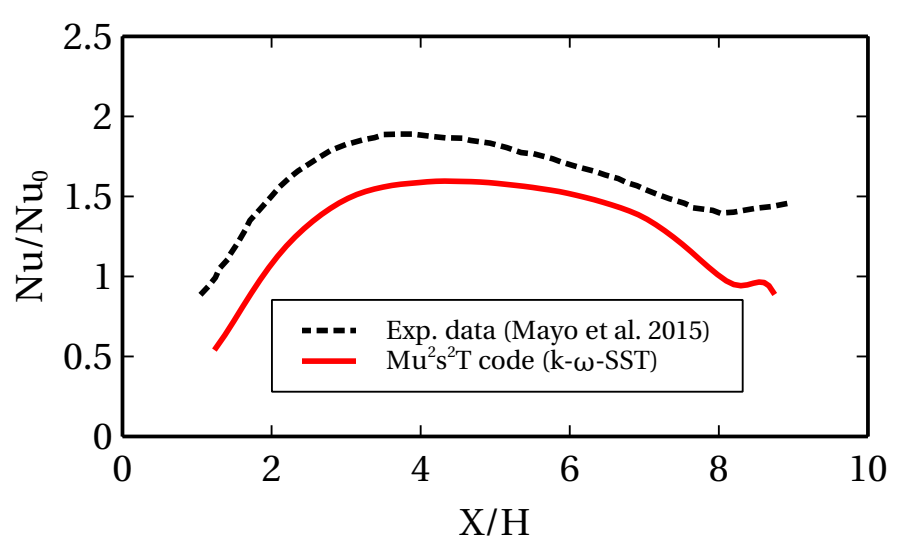

FIGURE 4. SPANWISE AVERAGED NU/NU DISTRIBUTION IN A RIBBED CHANNEL [32]

a proper turbulence model can provide reasonable predictions of heat transfer performance $[22,33,34,35,36]$. In this study, CFD simulations have been conducted to cover the whole test matrix (Table 1) to validate the numerical results, and improve the understanding of experimental results using flow-field information that is lacking in this complex test.

\section{RANS Code}

An in-house density-based RANS solver known as $M u^{2} s^{2} T$ $[37,38]$ has been used to conduct internal cooling studies. The code solves the 3D RANS equations using an edge-based data structure to spatially discretise the equations using a MUSCL scheme. Low Mach preconditioning is used to enhance the convergence and accuracy for low Mach number flows. The code is fully parallelized using MPI and executed on GPUs to improve computational efficiency.

In order to provide the readers an overall idea about which is the level of accuracy that can be expected from the simulations, the comparison with two well established rib roughened internal cooling cases $[31,32]$ have been included. The results are displayed in Fig. 3 and 4. It can first be noticed that the results of $M u^{2} s^{2} T$ code compared well with a third-party commercial code. In terms of heat transfer performance, the error in the spanwise averaged heat transfer coefficient in the inter-rib region can be seen in Fig. 4. The error is about $10 \%-20 \%$ in overall but the streamwise distribution is correct. These results give confidence to address more complex simulations.

\section{Computational Setup}

Previous studies $[16,17]$ have shown that, the entry shape can have a significant impact on the flow field and heat transfer performance. Therefore, the computational domain was extended to 


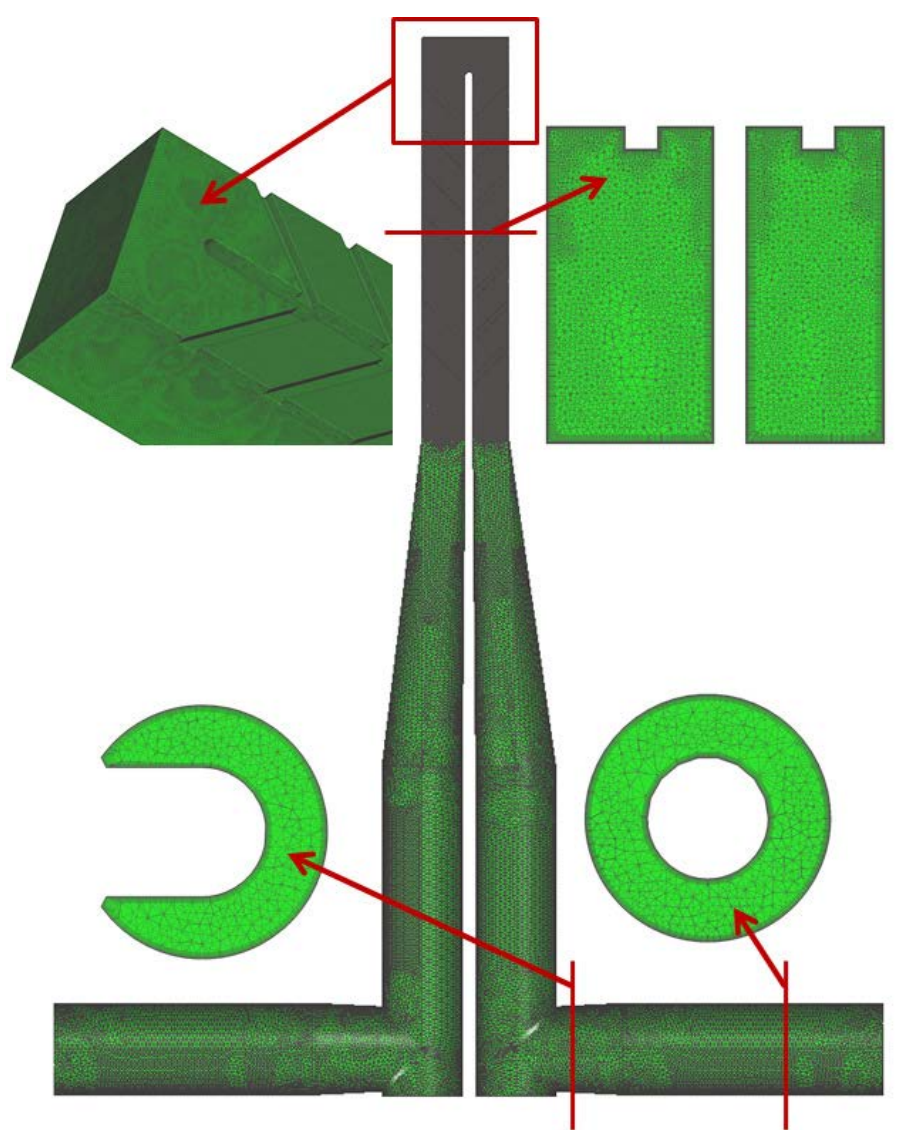

FIGURE 5. HYBRID MESH OF COMPUTATIONAL DOMAIN

include the supply system in order to account for inlet entry effects. Figure 5 shows the computational domain and mesh used in the numerical simulations. The inlet/outlet supply system has a circular pipe shape that transitions to a $\mathrm{C}$-shaped pipe near the joint with the transition pipe. The model is discretized by means of unstructured hybrid grids using an in-house mesh generator. The grid consists of a near-wall prismatic layer region, while the rest of the domain is discretized using isotropic tetrahedral elements. In total, 18 prismatic layers are constructed with $y^{+}<1$ for all the tested Reynolds numbers. The total grid size is 13.5 million cells. A sensitivity study was performed with a 8 million cell model, and the change in the section averaged heat transfer coefficient was less than 3\%. Hence, the solution obtained in current study is considered grid independent to our in-house code.

Steady-state simulations have been conducted using the $k-\omega$ SST turbulence model [39] with a inlet turbulence intensity of $5 \%$. Wall temperature distributions obtained from experimental data have been imposed in the heated wall instead of a constant temperature in order to remove the impact of wall heating conditions and maintain the same buoyancy effects than in the ex-

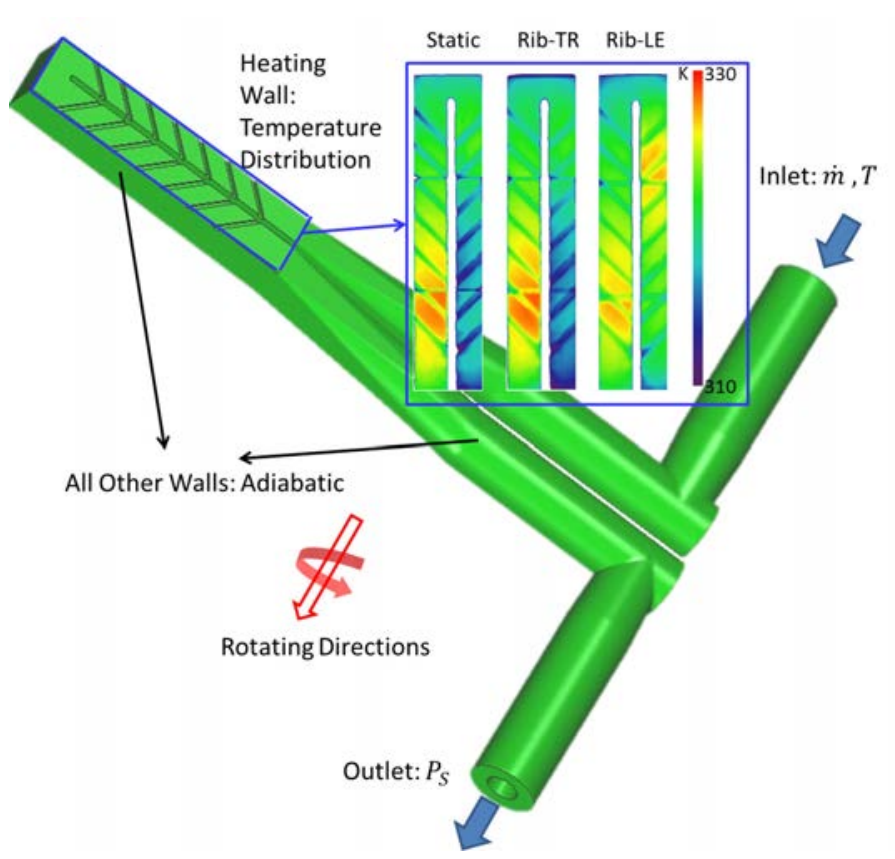

FIGURE 6. CFD BOUNDARY CONDITIONS SETUP

periment. The rest of the walls are considered as adiabatic. The inlet/outlet boundary conditions have been obtained from experimental measurements. Temperature and mass flow rate are provided at the inlet, and static pressure is set at outlet. In total 9 CFD simulations have been performed to cover the whole experimental test matrix.

\section{RESULTS AND DISCUSSION}

In the present study, the heat transfer behavior in a ribbed varying aspect ratio two-pass rectangular channel has been experimentally and numerically investigated. The averaged heat transfer performance of experimental data and corresponding numerical validations are presented. Then, as the complement of experiment, the details of heat transfer distribution and fluid flow are briefly discussed using the results of numerical simulations.

\section{Segment averaged heat transfer}

In order to quantify the heat transfer performance in cooling channels, the normalized segment averaged Nusselt number based on the smooth area between ribs is presented. The segment index notation is illustrated in Fig. 7.

Figure 8 shows the comparison between experimental and numerical results at $R e=15,000$. In the upstream passage, the segment averaged heat transfer data in stationary and ribbed surface rotating as trailing wall case (Rib-TR) are well predicted. Both experiment and numerical results show a nearly periodic 


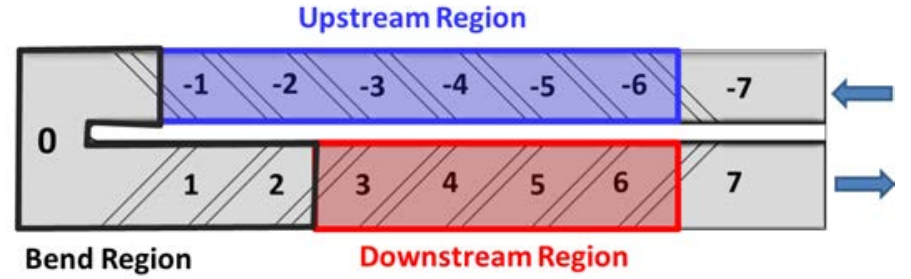

FIGURE 7. SEGMENT INDEX NOTATION AND REGION DECOMPOSITION

behavior in terms of averaged heat transfer. For ribbed surface as leading wall case (Rib-LE), the numerical results over-predict the negative effect of rotation in the first passage, underestimating the heat transfer in overal by about $30-40 \%$ when compared with the experimental data. This is because the rotation stabilizes the fluid at leading wall and enlarge the separation zone, especially when the wall is heated [40]. In this case, the local flow velocity and viscosity are very low, leading to hard predictions by RANS. In bend region, the heat transfer efficiency was over-predicted in all the numerical simulations. This maybe due to the fact that $k-\omega \mathrm{SST}$ model is not performing very well in impingement dominated flow and large separation areas.

The most important result is that, on the contrary than in previous works [21, 25, 17], the heat transfer efficiency drops, in ribbed trailing wall (Rib-TR) case compared to stationary case. This behavior is consistent, both in the experiments and in the numerical simulations. Moreover the magnitude of the drop in the upstream passage of ribbed trailing wall case is very large, being about $25 \%-30 \%$. This variation is much larger than the uncertainties that could be reasonably associated to either the experimental or numerical techniques used here.

In downstream region, the overall heat transfer performance is reduced because of the flow is slowed down by the increase of channel area. In the stationary case, the numerical simulation under-predicts the heat transfer by about $15 \%-20 \%$, but the trend was correctly captured. The Rib-TR case gives a good overall agreement. The experimental data show that in downstream region, the impact of the sense of rotation in the heat transfer coefficient is quite small, leading to a similar heat transfer levels in the two rotating cases.

Figure 9 presents the effect of rotation at $\operatorname{Re}=25,000$. It can be seen that the results are qualitatively similar to those of the $\mathrm{Re}=15,000$ cases. In ribbed leading wall (Rib-LE) case, the heat transfer is under-predicted in the upstream region and overpredicted in the bend region. The numerical simulations provide reasonable predictions of the heat transfer and its evolution along the channel for the stationary and ribbed trailing wall (Rib-TR) cases.

Due to restrictions of experimental facility, a different Rotation

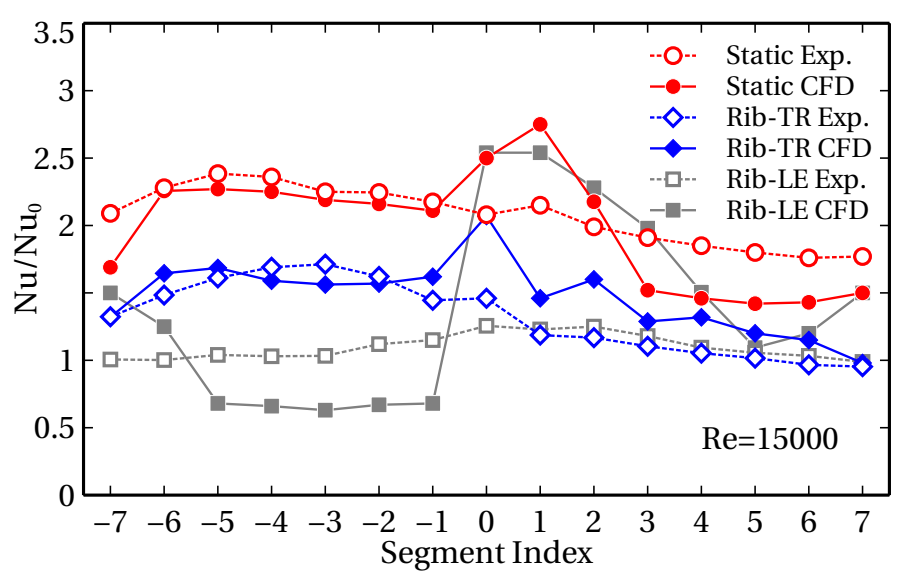

FIGURE 8. NORMALIZED SEGMENT AVERAGED NUSSELT NUMBER: EXP (FILlED SYMBOLS). VS CFD (SOLID SYMBOLS), $R e=15,000$, Ro $=0, \pm 0.3$

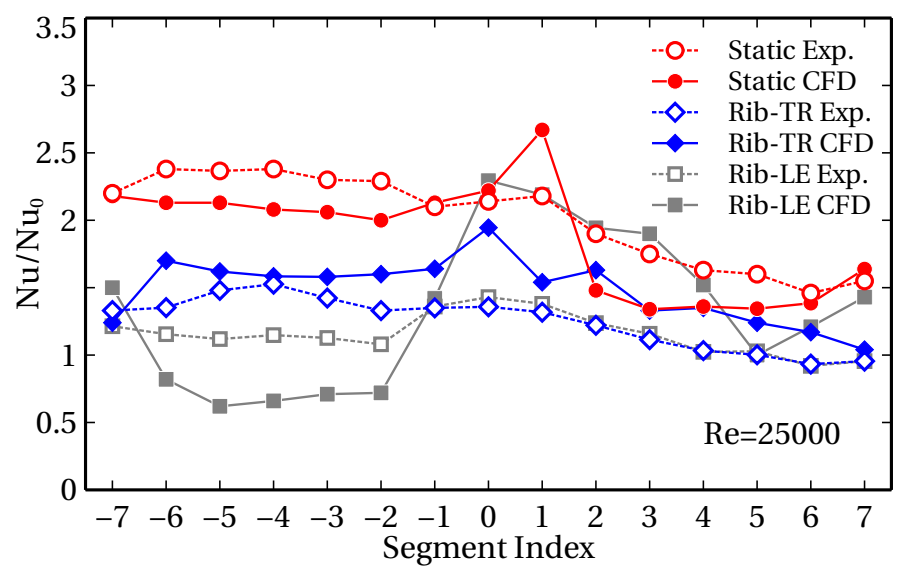

FIGURE 9. NORMALIZED SEGMENT AVERAGED NUSSELT NUMBER: EXP (FILLED SYMBOLS). VS CFD (SOLID SYMBOLS), $\mathrm{Re}=25,000, \mathrm{Ro}=0, \pm 0.3$

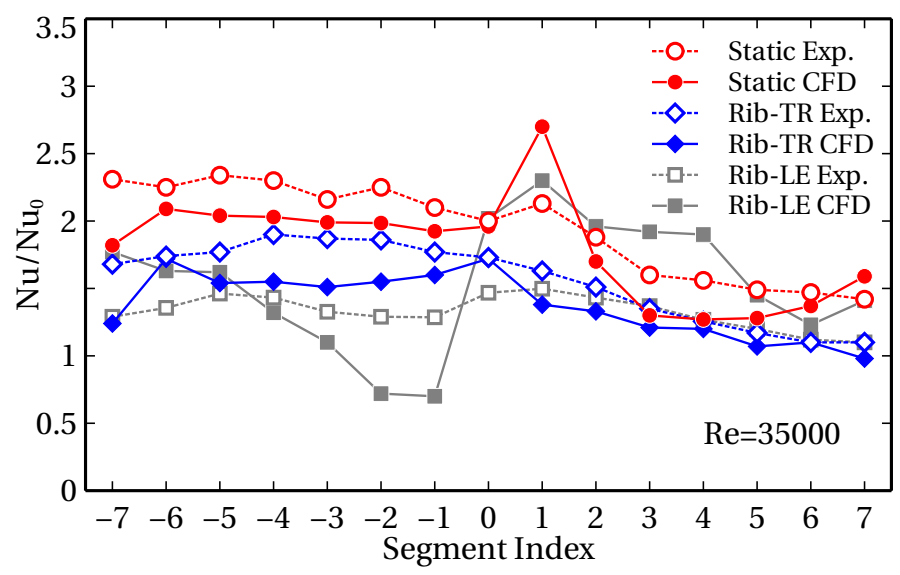

FIGURE 10. NORMALIZED SEGMENT AVERAGED NUSSELT NUMBER: EXP. (FILLED) VS CFD (SOLID), RE $=35000, \mathrm{Ro}=0, \pm 0.22$ 
number $(\mathrm{Ro}= \pm 0.22)$ was tested in $\mathrm{Re}=35,000$ rotating cases. As a result, in Fig. 10, the differences between stationary and rotations were reduced compared to the other two Reynolds numbers. In the stationary and ribbed trailing wall (Rib-TR) cases, the differences at the upstream regions becomes slightly larger than for the other Reynolds numbers, whereas for the ribbed leading wall (Rib-LE) case, the largest differences of prediction remains appearing close to bend region.

The numerical results in general are aligned with the experimental data, especially in stationary and ribbed trailing wall (Rib-TR) cases. Numerical results tend to over-predict heat transfer near the tip-bend regions and the separation regions after the bend in all three rotating conditions due to the fact that $k-\omega$ SST model may not perform very well in simulating impingement flow and large separation flow areas. In upstream channel of ribbed leading wall cases, in general the heat transfer was under-predicted by $25 \%$, indicating that RANS with $k-\omega$ SST may not provide good agreement in very low velocity and large separation areas.

\section{Influence of Reynolds number}

In addition, the region based consolidated heat transfer variation with Reynolds number is presented. The test channel has been split into three parts as shown in Fig. 7; the upstream region (segments -6 to -1), the bend influenced region (segments 0 to 2), and the downstream region (segments 3 to 6). The corresponding results are presented in Fig. 11 - 13. In the upstream passage, the experimental data show that the variation of heat transfer enhancement with $R e$ is very small for the stationary case. Numerical results are well predicted in heat transfer level, but with a slightly decreasing trend. The heat transfer level slightly decreases in ribbed trailing wall case. Conversely, in ribbed leading wall case, higher stream velocity increases the mixing with the large separated area, as a result, the heat transfer level is slightly increased with $R e$ under the same rotation number. The numerical simulations captured the overall trend correctly in ribbed leading wall (Rib-LE) case, but with under-predicted heat transfer. However, the variation in numerical ribbed trailing wall (Rib-TR) case is not significant compared against experimental data. All rotating cases at $\mathrm{Re}=35,000$ show that when reducing the Rotation number, the heat transfers are moving towards to the stationary case, as expected.

Figure 12 shows the results in the bend influenced region. The experimental data show that the influence of Reynolds number variation is quite small for all three cases under the same Rotation number. This is the combined result of the fact the $R e$ influence in the upstream region is small and that the effective of secondary structures in the bend region is large. The ribbed leading wall (Rib-LE) case has the largest difference between experimental data and numerical predictions. The numerical results of Rib-LE case shows similar level of enhancement as the stationary case

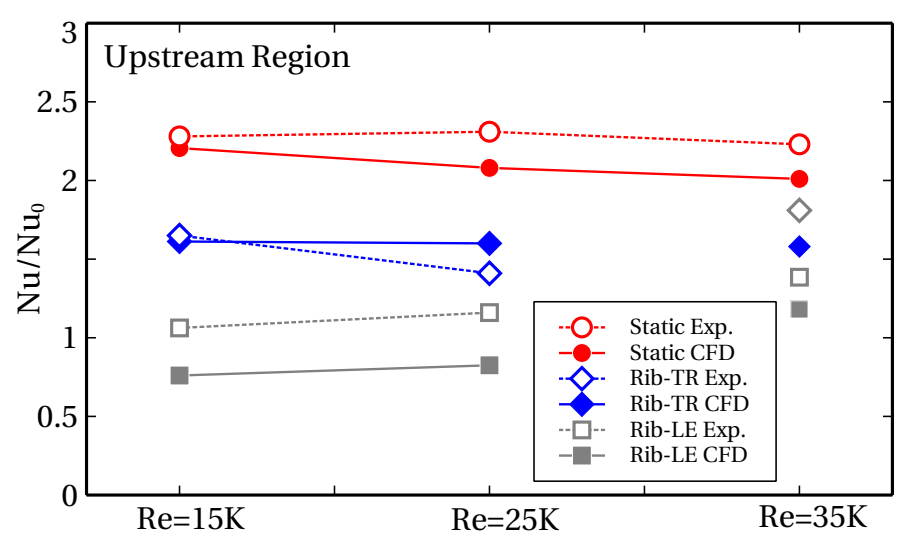

FIGURE 11. NORMALIZED AVERAGE NUSSELT NUMBER IN UPSTREAM REGIONS

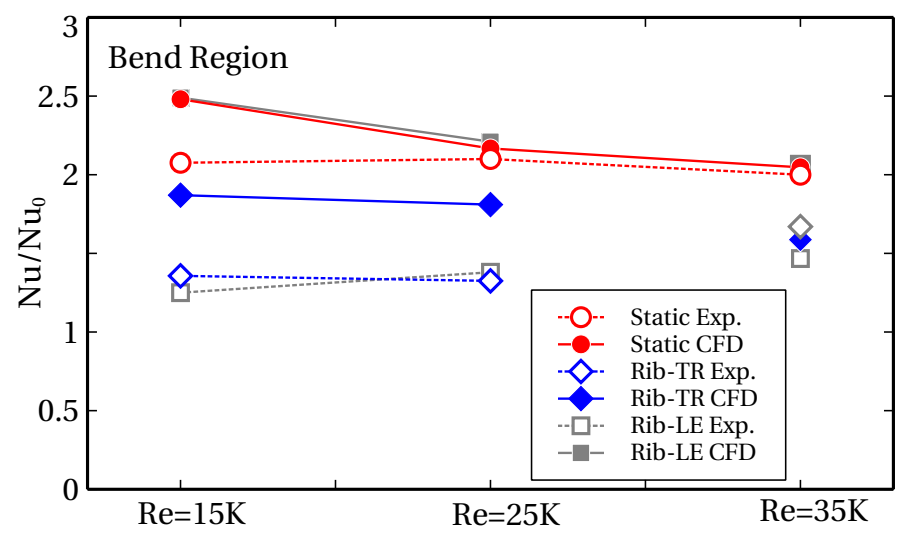

FIGURE 12. NORMALIZED AVERAGE NUSSELT NUMBER IN THE BEND REGION

rather than Rib-TR case.

The downstream passage results are presented in Fig. 13. the stationary case shows a slightly reduced heat transfer when Reynolds number increases. This is because at higher $R e$, the heat transfer level is high due to higher flow velocity, thus the effective of rib promoted enhancement drops. The numerical results predict a similar trend. Experimental data also show that rotation effects are nearly negligible due to the combination effect of centrifugal forces and Coriolis forces acting in contract directions in secondary passage. Numerical results also support similar conclusions.

\section{Detailed heat transfer distributions: rotation effect}

Figure 14 shows the contour plots of the normalized Nusselt number distributions of different Rotation numbers at $\mathrm{Re}=25,000$ obtained from the numerical simulations. The main objective is 


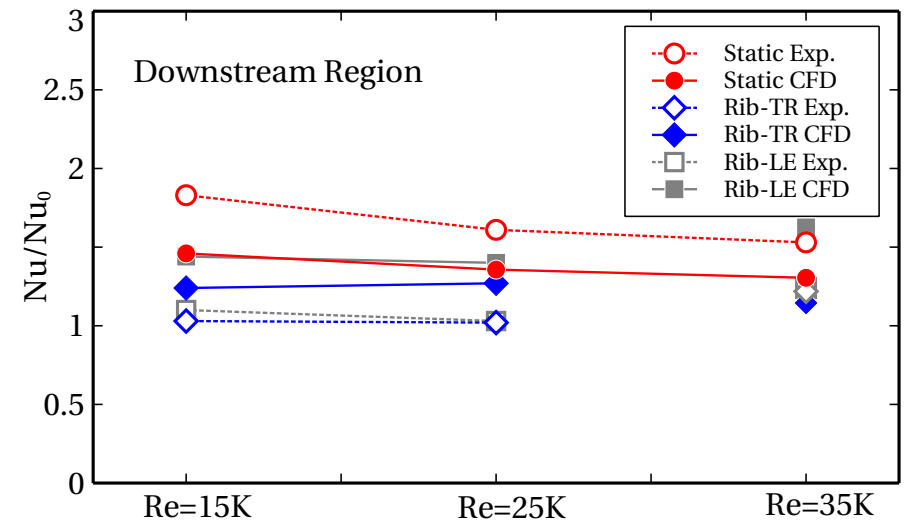

FIGURE 13. NORMALIZED AVERAGE NUSSELT NUMBER IN THE DOWNSTREAM PASSAGE

to complement experimental data and enhance the understanding of heat transfer details.

At the upstream passage of stationary case, a typical rib-induced heat transfer coefficient pattern is repeated from rib to rib. The heat transfer peak appears at the flow re-attachment region close to inner side wall. Then it develops toward to the outer side wall due to the secondary flow induced by the inclined ribs. This secondary flow pattern is clearly visualized in Fig. 15 (yellow line cut in Fig. 14 ). In ribbed trailing wall (Rib-TR) case, the ribbed surface acts as pressure side of rotation, leading to a rotationally induced secondary flow moving from the smooth to the ribbed side of the channel. As shown in Fig. 15, this secondary flow interacts with the rib induced secondary flow altering the flow field near the ribbed surface, resulting in a displacement of enhanced heat transfer region towards to the center of the the smooth area, as well as leading to a more uniform distribution compared to stationary cases. The heat transfer also shows a repeating patterns in upstream passage. In ribbed leading wall (Rib-LE) case, the rotation induced secondary flow moved away from ribbed surface, leading to reduced heat transfer performance in the upstream passage.

As mentioned in the previous section, a notable finding in this study is that the heat transfer was not enhanced in ribbed trailing wall (Rib-TR) case compared to stationary case. This unusual behavior maybe particularly explained in Fig. 16 to 18. Figure 16 displays the streamlines and relative velocity contour at the mid-plane of segment No. -3 ( red line cut in Fig. 14). The stationary case re-attaches faster than the Rib-TR case and the near wall flow has significantly higher velocity than the Rib-TR case leading to a higher heat transfer efficiency. Figures. 17 and 18 display the streamwise velocity and spanwise transverse velocity fields at the cutting plane. It can be seen that the streamwise velocity field in stationary case has similar shape as in RibTR case with slightly higher magnitude. However, as shown in

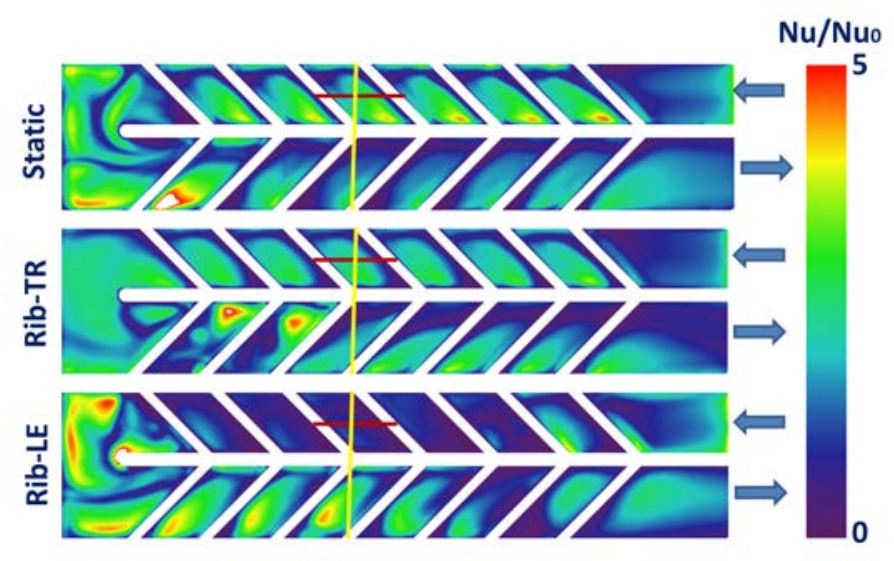

FIGURE 14. NUMERICAL RESULTS OF $N u / N u_{0}$ DISTRIBUTION AT $R e=25,000, \mathrm{Ro}=0,0.3,-0.3$

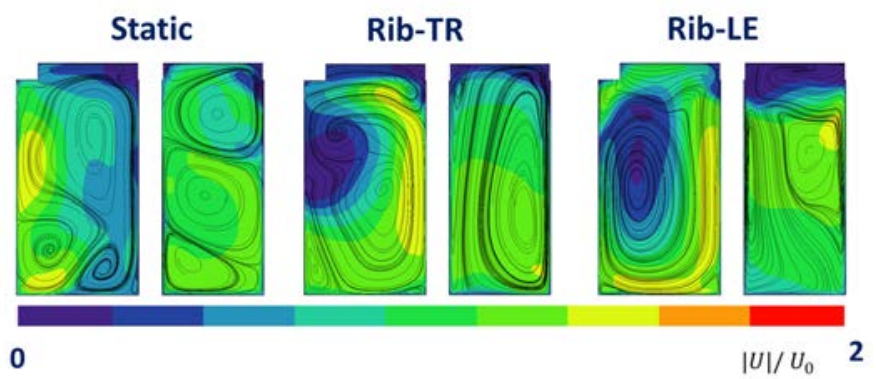

FIGURE 15. SECONDARY FLOW CONTOURS AT SEGMENT NO. - 3 , AT $R e=25,000, \mathrm{Ro}=0,0.3,-0.3$

Fig.18, the spanwise transverse velocity is significantly higher than in the Rib-TR case. The spanwise flow in stationary case is promoted by the inclined ribs. This secondary flow increases the momentum of fluid near the wall, promoting strong mixing and faster flow re-attachment. However, in Rib-TR case, the interaction between Coriolis force promoted secondary flow, and rib promoted secondary flow reduced the magnitude of spanwise transverse velocity. As a result, the heat transfer level in ribbed trailing wall case was not enhanced as generally observed.

Figure 18 displays the relative velocity profiles at mid-plane for the whole the channel height. It is shown that a very high velocity appears near the wall in stationary case. Furthermore, the main stream profiles show large differences from case to case. The particular entry geometry of the mock-up can alter the shape of main flow, resulting in a re-distribution of flow pattern, which may also be a reason of this unusual heat transfer behavior.

In ribbed leading wall case, the shear layer generated by the rib is stabilized by rotation and the separation bubble entrainment of main stream flow is strongly reduced. As can be seen in Fig. 


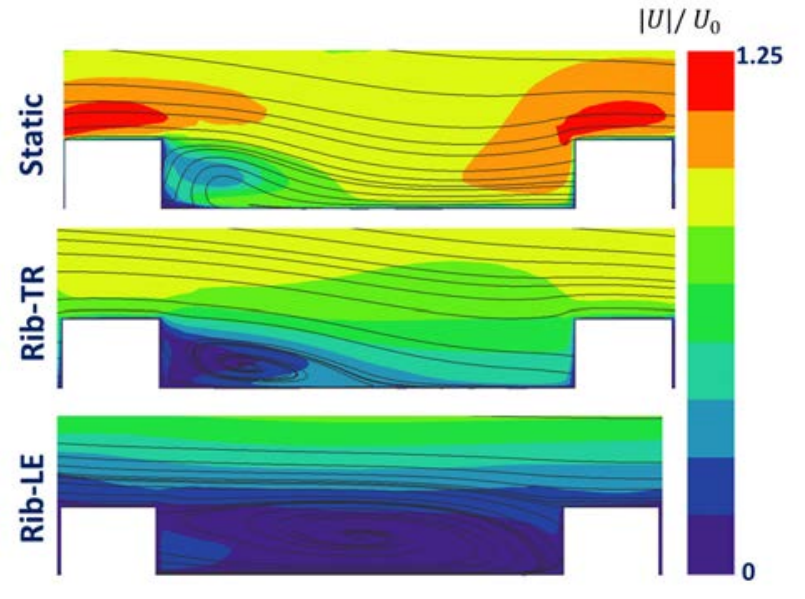

FIGURE 16. MODULUS OF THE VELOCITY FIELD AT MID PLANE OF SEGMENT NO. -3 , AT $R e=25,000, \mathrm{Ro}=0,0.3,-0.3$

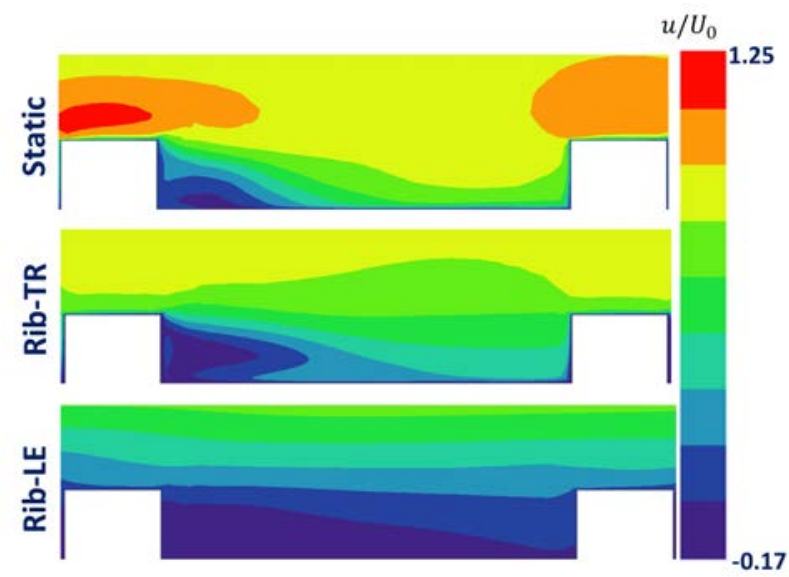

FIGURE 17. STREAM-WISE VELOCITY FIELD AT MID PLANE OF SEGMENT NO. -3 , AT $R e=25,000, \mathrm{Ro}=0,0.3,-0.3$

16, the low momentum separation bubble dominates the whole inter-rib region, and, as a result, the wall heat transfer is very low in this case. In addition, the ribbed leading wall (Rib-LE) case has small negative spanwise transverse velocity which indicates that the secondary flow has opposite direction as in stationary and ribbed trailing wall (Rib-TR) case (Fig. 18).

The flow field in bend region can be seen in details in Fig. 20. In stationary case, the heat transfer performance is greatly enhanced due to the increased fluid mixing at this region, led by the combined interaction of ribbed-induced secondary flow, impingement secondary vortex and Dean vortex induced by 180 degree sharp turn. A recirculation zone was found on the downstream side of the divider wall. Hence the effective cross sectional area for the main flow is reduced, resulting in a locally

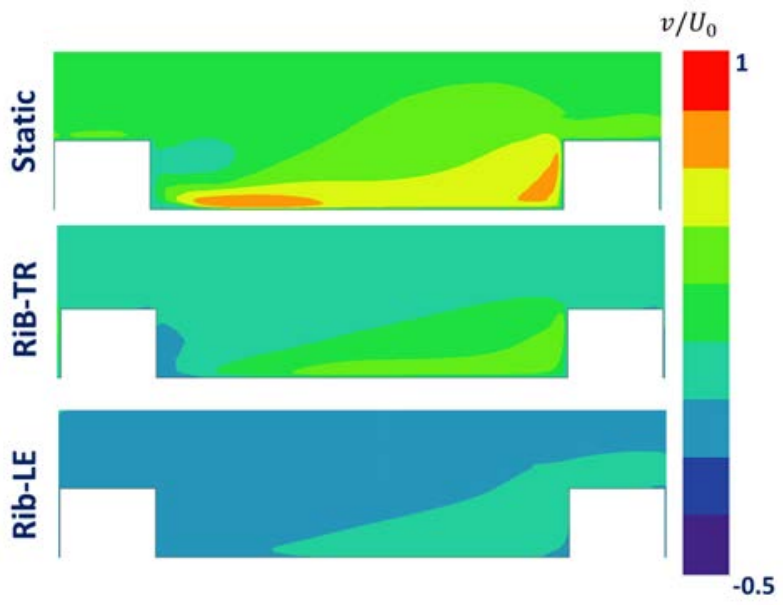

FIGURE 18. TRANSVERSE VELOCITY FIELD AT MID-PLANE OF SEGMENT “-3” WITH $R e=25000$, Ro=0, 0.3, -0.3

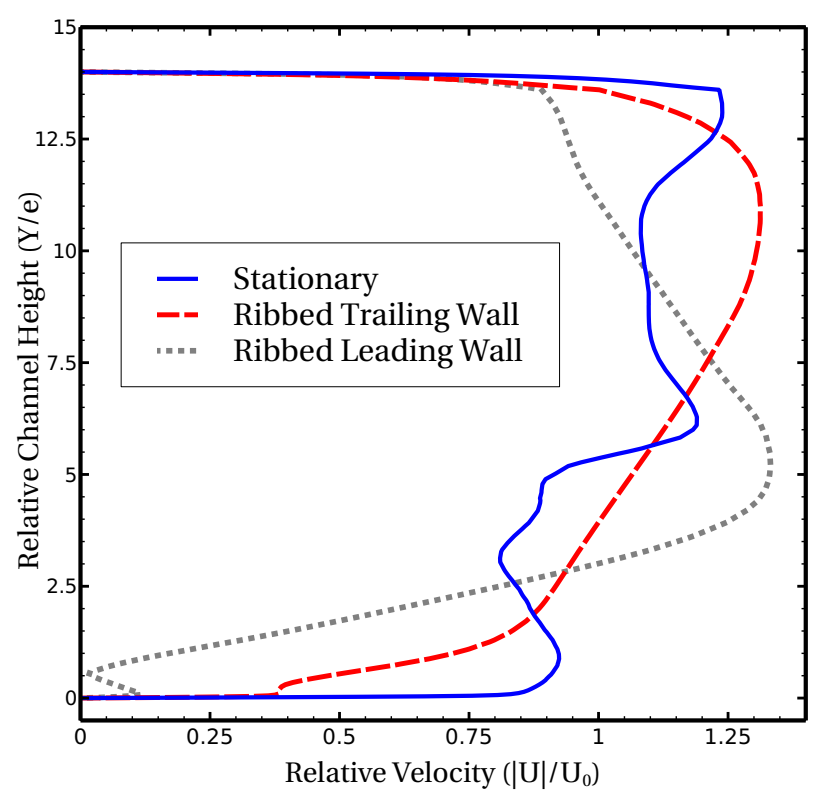

FIGURE 19. RELATIVE VELOCITY PROFILE AT THE MID-PLANE OF SEGMENT NO. -3 AT $R e=25000, R o=0,0.3,-0.3$

accelerated flow. In combination with the impingement of secondary flow in the downstream passage, this led to a very high heat transfer enhancement in the outer wall of the segment after the bend (Fig. 14). Then, the influence of the bend rapidly decays downstream and disappears after about 2 to 3 ribs. A repeating heat transfer pattern appears again further downstream. However, due to the area increase, the flow is slow down at outlet passage resulting in a smaller heat transfer coefficient than in the inlet passage. 


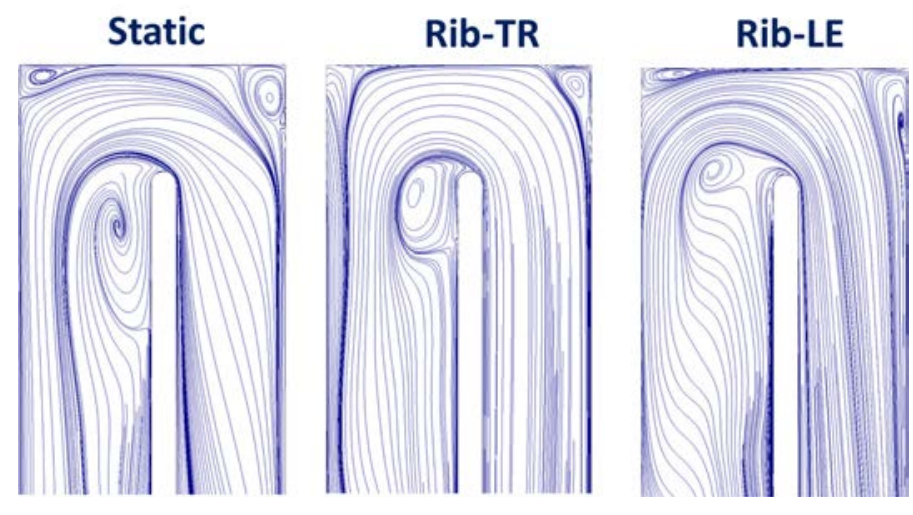

FIGURE 20. FLOW FIELD IN BEND REGION AT $R e=25,000$, WITH Ro $=0,0.3,-0.3$

In ribbed trailing wall case, the heat transfer at the bend region shows more uniform behavior compared to stationary case (Fig. 14). This phenomenon was due to rotating induced secondary flow in the bend region smooths the heat transfer distribution. In addition, a smaller recirculation zone was observed after the bend divider wall. This faster reattachment weaken the accelerated flow after the bend and resulted in a heat transfer peak moved to inner side wall after bend. After about 2-3 segment lengths, a repeating heat transfer pattern appears again, with a different distribution, as in stationary cases.

In ribbed leading wall case, the shape of recirculation zone is similar but a little larger than in the stationary case. A strong accelerated flow after bend is also shown. Therefore, heat transfer also shows similar contour and magnitude as in stationary. However, no such large enhancement was observed in experiment.

\section{Evolution of secondary flow}

Flow field in two-pass ribbed channels is very complex, threedimensional, and affected by inlet flow distribution, rib and rotation induced secondary flow, and sharp turns. Figure 21 shows the evolution of secondary flow pattern at different cutting sections of the channel at $\mathrm{Re}=25,000$. Plane 1 represents the secondary flow pattern at the inlet of the passage. It is clearly seen that the entry geometry formed a counter clockwise vortex at the middle of the channel in the stationary case. It is observed that, in planes 2,3 and 4, this central vortex is combined with the clockwise rib-induced vortex, which is dominating at the upper part of the channel. Meanwhile, a much weaker rotating vortex is developed at the bottom of the channel. Plane 5 shows the secondary flow pattern at the bend center plane. Typical shape of Dean vortex is visualized. In this region, fluid flow impinging in the tip of the channel and forming a radial pressure gradient with high static pressure near the tip wall. Near the top and bottom walls, where the fluid motion was slow, the pressure gradient is not balanced, therefore a clockwise circulation appears at the top and a counter-clockwise circulation appears at the bottom. In this case, the circulation near upper wall was amplified due to the rib-induced secondary flow has a same direction. Meanwhile, the trace of inlet shape formed circulation can still be seen near the center.

In ribbed trailing wall (Rib-TR) case, rotating induced a secondary flow moving fluid from bottom to the top of the channel. At the side wall, the fluid was moving slow, the pressure gradient led to a contrary flow from upper side to bottom to close the loop. In addition, the rotating induced secondary flow interact with rib induced secondary flow resulting in a completely different flow pattern. The development of the flow pattern can be seen from plane 1 to plane 4 . In the bend center plane (plane 5), the Dean vortex at the upper part of the channel decayed due to the influence of rotation effect, this result in a more uniform heat transfer at the bend region. (Fig. 14)

In ribbed leading wall (Rib-LE) case, opposite behavior was shown due to the change of rotating direction. The rotating induced circulation acts in difference direction as the rib-induced secondary flow. This mixing significantly weaken the heat transfer on the ribbed wall. In the bend center plane, the form of Dean vortex expressed contrary behavior as in ribbed trailing wall (Rib-TR) case. The bottom vortex decayed, while a clear pattern can be seen at the upper part. This phenomenon resulted in a similar heat transfer pattern at bend region compared to stationary case (Fig. 14)

\section{CONCLUSIONS}

This paper studies experimentally and numerically the heat transfer behavior of an engine representative varying aspect ratio twopass channel under rotation. In addition, heat transfer distribution and secondary flow pattern have been briefly discussed.

This set of experiments provide a heat transfer data base and overall understanding of the heat transfer performance that can be expected in a realistic configuration which has rarely been studied in literature. Numerical simulations provide reasonable predictions in terms of averaged heat transfer performance and which is more important from an engineering point of view, similar trends than those obtained in the experimental campaign, both in stationary and ribbed trailing wall rotation cases in the whole range of tested Reynolds numbers. Although large differences are observed in under-predicting heat transfer in first passage of ribbed leading wall rotation cases, and over-predicting in all cases near the bend, where the heat transfer can be $30 \%-60 \%$ differ from experiment. This possibly due to the limitations of RANS with $k-\omega \mathrm{SST}$ in heat transfer prediction at very low velocity with large separation areas and impingement flow dominated areas. The experimental data show that due to the centrifugal and buoyancy forces acting in opposite manner in down- 


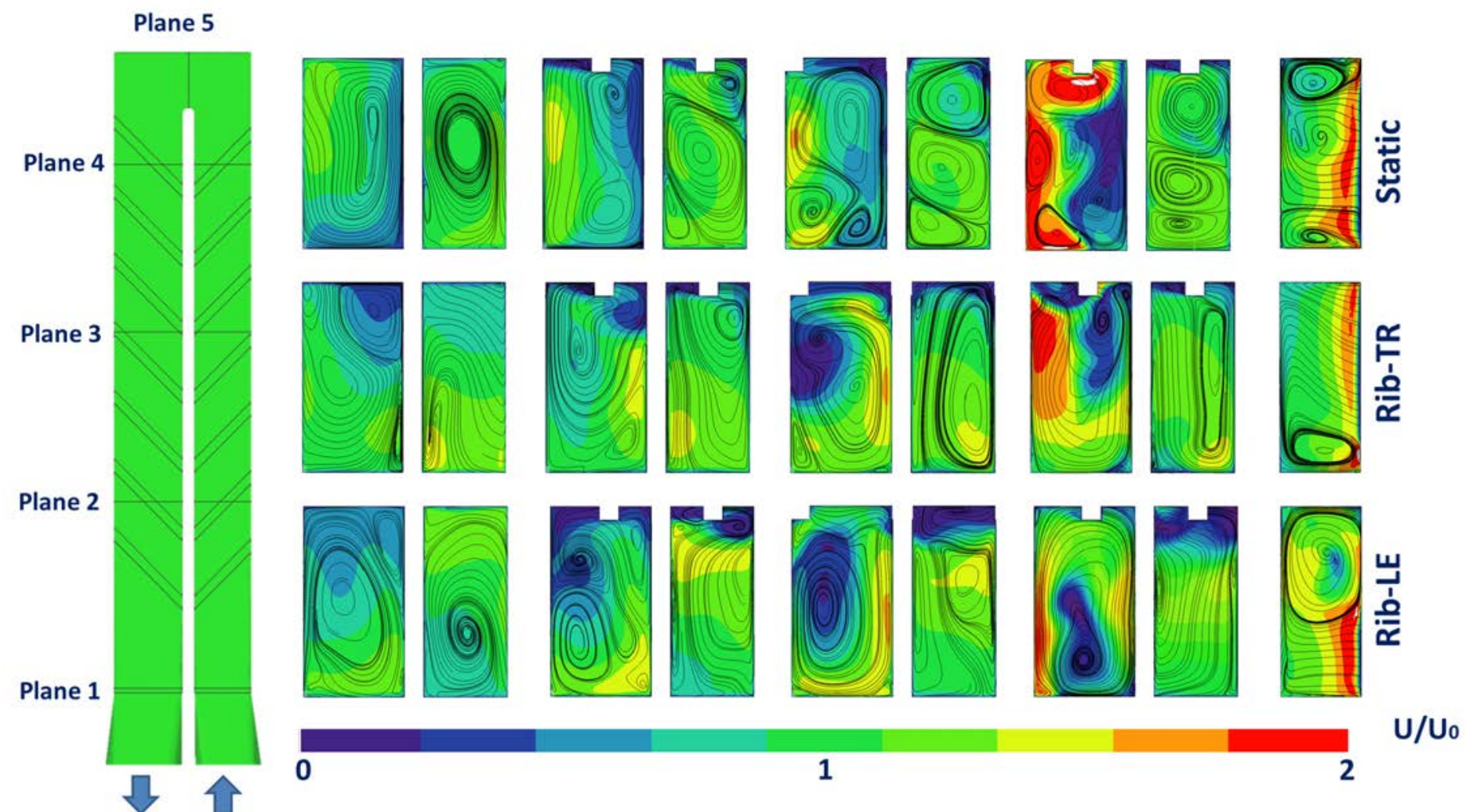

FIGURE 21. SECONDARY FLOWS AT DIFFERENT CUTTING PLANES (Re=25K)

stream passage, the differences in heat transfer level for the two rotating cases are minimal.

The most important finding was that the heat transfer in ribbed trailing wall cases was not enhanced compared to stationary cases. This behavior has been shown agreement both by experiment and numerical predictions. This may be due to the fact that the spanwise velocity has been weakened by the interaction of rotation induced secondary flow and rib induced secondary flow in this particular case. Another possible reason could be the complex entry shape results in a variation of main flow, leading to a re-distributed velocity profile near the wall. Whatever the case is, this behavior is reproduced by RANS simulations, which is very important from a practical point of view.

Upon the study of flow-field, it can be concluded that rotation significantly changes the secondary flow pattern inside the channel. Special interest was payed in the center plane of the bend, where the shape of Dean vortex was transformed due to the effect of rotation. This fact leads to different heat transfer distribution at the bend region of the channel. Moreover, rotation effects also alter the flow reattachment after the bend, resulting in a very complex flow pattern in the downstream passage.

This study also concludes that a RANS approach using $k-\omega$ SST model can be considered satisfactory in overall heat transfer predictions, taking into consideration the complexity of the flow created by the two-pass ribbed channel.

\section{ACKNOWLEDGMENTS}

The research leading to these results has received funding from the European Union Seventh Framework Programme (FP7/20072013) under grant agreement No. 233799 (ERICKA). The authors wish to thank Industria de Turbopropulsores S.A. (ITP) for its technical support and computing resource. Thank Laura Benet from SNECMA for providing the design data of the cooling channel. The ERICKA consortium and members, in particular ITP, ONERA, and SNECMA, are greatly acknowledged for allowing the authors to publish this paper.

\section{References}

[1] Han, J., 1988. "Heat transfer and friction characteristics in rectangular channels with rib tabulators". Journal of Heat Transfer, 1, pp. 321-328.

[2] Han, J., and Park, J. S., 1988. "Developing heat transfer in rectangular channels with rib turbulators". International Journal of Heat and Mass Transfer, 31(1), pp. 183-195. 
[3] Park, J., Han, J., Huang, Y., Ou, S., and Boyle, R., 1992. "Heat transfer performance comparisons of five different rectangular channels with parallel angled ribs". International Journal of Heat and Mass Transfer, 35(11), pp. 2891-2903.

[4] Taslim, M., and Wadsworth, C., 1997. "An experimental investigation of the rib surface-averaged heat transfer coefficient in a rib-roughened square passage". Journal of Turbomachinery, 119(2), pp. 381-389.

[5] Taslim, M., and Lengkong, A., 1998. "45 deg staggered rib heat transfer coefficient measurements in a square channel". Journal of Turbomachinery, 120(3), pp. 571-580.

[6] Taslim, M., and Korotky, G., 1998. "Low-aspect-ratio rib heat transfer coefficient measurements in a square channel”. Journal of Turbomachinery, 120, pp. 831-838.

[7] Wagner, J., Johnson, B., and Hajek, T., 1991. "Heat transfer in rotating passages with smooth walls and radial outward flow". Journal of Turbomachinery, 113(1), pp. 42-51.

[8] Wagner, J., Johnson, B., and Kopper, F., 1991. "Heat transfer in rotating serpentine passages with smooth walls". Journal of Turbomachinery, 113(3), pp. 321-330.

[9] Johnson, B., Wagner, J., Steuber, G., and Yeh, F., 1994. "Heat transfer in rotating serpentine passages with trips skewed to the flow". Journal of Turbomachinery, 116(1), pp. 113-123.

[10] Dutta, S., and Han, J.-C., 1996. "Local heat transfer in rotating smooth and ribbed two-pass square channels with three channel orientations". Journal of Heat Transfer, 118(3), pp. 578-584.

[11] Al-Hadhrami, L., and Han, J.-C., 2003. "Effect of rotation on heat transfer in two-pass square channels with five different orientations of 45 angled rib turbulators". International Journal of Heat and Mass Transfer, 46(4), pp. 653669.

[12] Fu, W.-L., Wright, L. M., and Han, J.-C., 2005. "Buoyancy effects on heat transfer in five different aspect-ratio rectangular channels with smooth walls and 45-degree ribbed walls”. In ASME Turbo Expo 2005, American Society of Mechanical Engineers, pp. GT2005-68493.

[13] Fu, W.-L., Wright, L. M., and Han, J.-C., 2004. "Heat transfer in two-pass rotating rectangular channels (ar= 1: 2 and ar= 1: 4) with 45 angled rib turbulators". In ASME Turbo Expo 2004, American Society of Mechanical Engineers, pp. GT2004-53261.

[14] Iacovides, H., Kounadis, D., and Xu, Z., 2009. "Experimental study of thermal development in a rotating squareended u-bend". Experimental Thermal and Fluid Science, 33(3), pp. 482-494.

[15] Lucci, J. M., Amano, R., and Guntur, K., 2007. “Turbulent flow and heat transfer in variable geometry u-bend blade cooling passage". In ASME Turbo Expo 2007, American Society of Mechanical Engineers, pp. GT2007-27120.
[16] Wright, L. M., Fu, W.-L., and Han, J.-C., 2005. "Influence of entrance geometry on heat transfer in rotating rectangular cooling channels (ar= 4: 1) with angled ribs". Journal of Heat Transfer, 127(4), pp. 378-387.

[17] Huh, M., Lei, J., Liu, Y.-H., and Han, J.-C., 2011. "High rotation number effects on heat transfer in a rectangular (ar= 2: 1) two-pass channel". Journal of Turbomachinery, 133(2), p. 021001.

[18] Dawes, W., 2008. "Rapid prototyping design optimization using flow sculpting”. Journal of Turbomachinery, 130(3), May, p. 031012.

[19] Lin, Y.-L., Shih, T.-P., Stephens, M., and Chyu, M., 2001. "A numerical study of flow and heat transfer in a smooth and ribbed u-duct with and without rotation". Journal of Heat Transfer, 123(2), pp. 219-232.

[20] Al-Qahtani, M., Jang, Y.-J., Chen, H.-C., and Han, J.-C., 2002. "Prediction of flow and heat transfer in rotating two-pass rectangular channels with 45-deg rib turbulators". Journal of Turbomachinery, 124(2), pp. 242-250.

[21] Al-Qahtani, M., Chen, H.-C., and Han, J.-C., 2003. "A numerical study of flow and heat transfer in rotating rectangular channels ( $\operatorname{ar}=4)$ with $45 \mathrm{deg}$ rib turbulators by reynolds stress turbulence model". Journal of Heat Transfer, 125(1), pp. 19-26.

[22] Shevchuk, I. V., Jenkins, S. C., Weigand, B., von Wolfersdorf, J., Neumann, S. O., and Schnieder, M., 2011. "Validation and analysis of numerical results for a varying aspect ratio two-pass internal cooling channel". Journal of Heat Transfer, 133(5), p. 051701.

[23] Bonhoff, B., Tomm, U., Johnson, B. V., and Jennions, I., 1997. "Heat transfer predictions for rotating u-shaped coolant channels with skewed ribs and with smooth walls". In ASME Turbo Expo 1997, American Society of Mechanical Engineers, pp. 97-GT-162.

[24] Bonhoff, B., Parneix, S., Leusch, J., Johnson, B., Schabacker, J., and Bölcs, A., 1999. "Experimental and numerical study of developed flow and heat transfer in coolant channels with 45 degree ribs". International Journal of Heat and Fluid Flow, 20(3), pp. 311-319.

[25] Han, J.-C., and Chen, H.-C., 2006. "Turbine blade internal cooling passages with rib turbulators". Journal of Propulsion and Power, 22(2), pp. 226-248.

[26] Sewall, E. A., and Tafti, D. K., 2005. "Large eddy simulation of flow and heat transfer in the 180 bend region of a stationary ribbed gas turbine internal cooling duct". In ASME Turbo Expo 2005, American Society of Mechanical Engineers, pp. GT2005-68518.

[27] Abdel-Wahab, S., and Tafti, D. K., 2004. "Large eddy simulation of flow and heat transfer in a staggered 45 ribbed duct". In ASME Turbo Expo 2004, American Society of Mechanical Engineers, pp. GT2004-53800.

[28] Saha, A. K., and Acharya, S., 2005. "Flow and heat transfer 
in internally ribbed ducts with rotation: an assessment of les and rans". Journal of Turbomachinery, 127(2), pp. 306320.

[29] Fransen, R., Vial, L., and Gicquel, L. Y., 2013. "Large eddy simulation of rotating ribbed channel". In ASME Turbo Expo 2013, American Society of Mechanical Engineers, pp. GT2013-95076.

[30] Kays, W. M., Crawford, M. E., and Weigand, B., 2012. Convective heat and mass transfer. Tata McGraw-Hill Education.

[31] Rau, G., Cİ§akan, M., Moeller, D., and Arts, T., 1998. “The effect of periodic ribs on the local aerodynamic and heat transfer performance of a straight cooling channel". Journal of Turbomachinery, 120(2), pp. 368-375.

[32] Mayo, I., Lahalle, A., Gori, G. L., and Arts, T., 2015. "Aerothermal characterization of a rotating ribbed channel at engine representative conditions: Part ii-detailed lct measurements". In ASME Turbo Expo 2015, American Society of Mechanical Engineers, pp. GT2015-42847.

[33] Schüler, M., Zehnder, F., Weigand, B., von Wolfersdorf, J., and Neumann, S. O., 2011. "The effect of turning vanes on pressure loss and heat transfer of a ribbed rectangular twopass internal cooling channel". Journal of Turbomachinery, 133(2), p. 021017.

[34] Schüler, M., Dreher, H.-M., Neumann, S., Weigand, B., and Elfert, M., 2012. "Numerical predictions of the effect of rotation on fluid flow and heat transfer in an engine-similar two-pass internal cooling channel with smooth and ribbed walls". Journal of Turbomachinery, 134(2), p. 021021.

[35] Pape, D., Jeanmart, H., von Wolfersdorf, J., and Weigand, B., 2004. "Influence of the 180 bend geometry on the pressure loss and heat transfer in a high aspect ratio rectangular smooth channel". In ASME Turbo Expo 2004, American Society of Mechanical Engineers, pp. GT2004-53753.

[36] Su, G., Chen, H.-C., Han, J.-C., and Heidmann, J. D., 2004. "Computation of flow and heat transfer in two-pass rotating rectangular channels ( $\operatorname{ar}=1: 1, \operatorname{ar}=1: 2$, ar= 1: 4) with 45deg. angled ribs by reynolds stress turbulence model". In ASME Turbo Expo 2004, American Society of Mechanical Engineers, pp. GT2004-53662.

[37] Burgos, M. A., Contreras, J., and Corral, R., 2011. "Efficient edge-based rotor/stator interaction method". AIAA journal, 49(1), pp. 19-31.

[38] Gisbert, F., Corral, R., and Pastor, G., 2011. "Implementation of an edge-based navier-stokes solver for unstructured grids in graphics processing units". In ASME Turbo Expo 2011, American Society of Mechanical Engineers, pp. gt2011-46224.

[39] Menter, F. R., 1994. "Two-equation eddy-viscosity turbulence models for engineering applications". AIAA journal, 32(8), pp. 1598-1605.

[40] Coletti, F., Jacono, D. L., Cresci, I., and Arts, T., 2014.
"Turbulent flow in rib-roughened channel under the effect of coriolis and rotational buoyancy forces". Physics of Fluids, 26(4), p. 045111. 